\title{
ANÁLISIS DE SOBRETENSIONES RESULTANTES DE DESCARGAS ATMOSFÉRICAS PARA SUELOS DEPENDIENTES DE LA FRECUENCIA
}

\author{
ANALYSIS OF OVERVOLTAGES RESULTING FROM ATMOSPHERIC \\ DISCHARGES FOR FREQUENCY-DEPENDENT SOILS
}

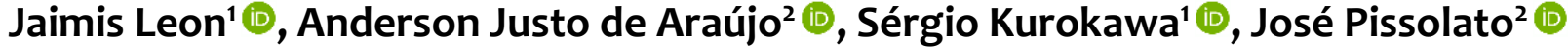

${ }^{1}$ Facultad de Ingeniería Eléctrica, Universidad Estadual Paulista "Júlio de Mesquita Filho", UNESP, Ilha Solteira-Brasil.

${ }^{2}$ Facultad de Ingeniería Eléctrica e Computación, Universidad de Campinas, UNICAMP, Campinas-Brasil.

*E-mail: jeimis.leon@gmail.com

Recibido (Received): 23/01/2020 Aceptado (Accepted): 11/ 08 / 2020

\begin{abstract}
RESUMEN
Este articulo presenta un estudio comparativo de la impedancia de un electrodo vertical enterrado en un suelo homogéneo donde los parámetros eléctricos (resistividad y permisibilidad) son dependientes de la frecuencia en relación con las impedancias obtenidas con los parámetros del suelo constantes. Este estudio, considera que el electrodo vertical se modela como una línea de transmisión (LT) corta cuyos parámetros eléctricos (resistencia, inductancia, capacitancia y conductancia) se distribuyen a lo largo de su longitud. De esta manera, la impedancia del electrodo se calcula como la impedancia armónica de la LT en función de la frecuencia. Una vez que se obtienen las impedancias de aterramiento del electrodo, y utilizando la técnica del Vector Fitting, circuitos eléctricos equivalentes son obtenidos directamente en el dominio del tiempo. La ventaja de usar este tipo de representación es que el circuito equivalente puede insertarse fácilmente en software de tipo EMTP, para realizar análisis de los transitorios electromagnéticos. De esta manera, se pueden calcular la elevación del potencial en el aterramiento para una descarga atmosférica incidente en el electrodo. Adicionalmente, se inyecta una descarga atmosférica en la parte superior de la torre de transmisión cuyo sistema de puesta a tierra está compuesto por un electrodo vertical, y se calculan las tensiones transitorias en los brazos y la base de la torre. Los resultados muestran que las tensiones se modifican significativamente por la presencia del suelo con parámetros dependientes de la frecuencia, especialmente en los picos, y que un análisis preciso de los transitorios electromagnéticos en los sistemas eléctricos debe tener en cuenta este fenómeno.
\end{abstract}

Palabras Clave: Transitorio electromagnético, Sistema de aterramiento, Impedancia de aterramiento, Descarga atmosférica, Parámetros del suelo.

\section{ABSTRACT}

This paper presents a comparative analysis of the electrode impedance for a vertical rod buried in a homogeneous ground where the soil electrical parameters (resistivity and permittivity) are frequency-dependent in relation to the impedances obtained with frequency-constant parameters. In this study, it is taken into account that vertical rod can be seen as a short transmission line whose electrical parameters (resistance, inductance, capacitance and conductance) are distributed along its length. Thus, the rod impedance is computed as being the harmonic impedance of the transmission line as a function of frequency. Once the rod impedances are obtained, and using the Vector Fitting technique, equivalent electrical circuits are obtained directly in time domain. The advantage of this type of representation is that equivalent circuit can be easily incorporated into EMTP-type programs for the electromagnetic transient analysis. In this model, the grounding potential rise (GPR) generated due to a lightning stroke at the rod can be calculated. Additionally, an impulsive current source, representing a lightning stroke, is injected at the top of a transmission tower whose the grounding system is composed by a vertical rod, and the transient voltages at the cross-arms and tower base are calculated. The results show that the voltages are significantly modified by the presence of the soil with frequency-dependent parameters, especially their voltage peaks, and that an accurate analysis of the transient in power systems must take this phenomenon into account.

Keywords: Electromagnetic transients, Grounding systems, Grounding impedance, Lightning stroke, Soil parameters.

\section{INTRODUCCION}

Los conductores de aterramiento son uno de los mecanismos para mitigar las sobretensiones causadas por descargas atmosféricas en las torres de transmisión. Estas sobretensiones pueden causar interrupciones en los sistemas de energía debido al back flashovers (BFs), si la impedancia conectada al pie de la torre no está diseñada correctamente [1]. Además, la impedancia de aterramiento se emplea para disipar las corrientes de fallas en el suelo, reducir 
el potencial de paso y toque alrededor de la torre o subestaciones eléctricas para protección del personal cercano y de los equipos que se conectan en los sistemas de energía eléctrica [2].

Para calcular las sobretensiones transitorias en los sistemas eléctricos, la impedancia de aterramiento suele ser representada por: (i) una resistencia concentrada o (ii) un circuito eléctrico equivalente, obtenido por aproximaciones casi-estáticas [3], [4]. Sin embargo, la impedancia de aterramiento depende de la frecuencia, donde en las bajas frecuencias presentan un comportamiento puramente resistivo. Entretanto, a partir de una dada frecuencia, los efectos capacitivos e inductivos son predominantes y no se pueden desconsiderar para el análisis de transitorio electromagnético. Además, las descargas atmosféricas son caracterizadas por un amplio espectro de frecuencia que varía de $\mathrm{DC}$ hasta decenas de $\mathrm{MHz}$ [5].

Otro aspecto muy importante es la dependencia de los parámetros eléctricos del suelo (permisividad y resistividad) con la frecuencia [6]. El suelo está compuesto por una composición muy compleja de materia orgánica, humedad y minerales que se compactan en camadas. De esta manera, los parámetros eléctricos resistividad y permisividad son fuertemente dependientes de la frecuencia debido a procesos complejos de polarización eléctrica, en cuanto que la permeabilidad magnética permanece prácticamente constante [7].

En este trabajo, las impedancias de aterramiento de un conductor vertical son calculadas considerando el electrodo como una línea de transmisión corta. En esta aproximación, se pueden considerar los parámetros eléctricos del suelo dependientes de la frecuencia o constantes. En seguida, se calcula la elevación de potencial (Ground Potential Rise - GPR) para una descarga atmosférica incidente en el electrodo. También, la misma descarga atmosférica se inyecta en la parte superior de una torre de transmisión que tiene en su base un sistema de aterramiento compuesto por un electrodo vertical. Son calculadas las tensiones producidas en los brazos de la estructura y el GPR en su base. Los resultados muestran que cuando se consideran el efecto de la frecuencia en los parámetros del suelo, las tensiones son significativamente reducidas en relación a las tensiones obtenidas con los parámetros constantes del suelo. Esa diferencia pronunciada puede sobreestimar los proyectos de aisladores y afectar la coordinación de asilamiento para protección de la línea de transmisión atingidas por las descargas atmosféricas y comprometer el funcionamiento del sistema eléctrico.

\section{MODELADO DEL SISTEMA DE ATERRAMIENTO USANDO LA TEORIA DE LINEA DE TRANSMISSIÓN}

Los electrodos cilíndricos verticales u horizontales pueden ser presentados por el modelo de línea de transmisión (MLT), donde los parámetros eléctricos (resistencia, inductancia, capacitancia y conductancia) de un conductor son distribuidos a lo largo de su longitud [8]. En la Fig. 1, se muestra un conductor vertical simple enterrado en un suelo homogéneo.

Donde $d$ es la profundidad y a es el radio de su sección transversal. Los parámetros del suelo son: resistividad $\rho_{s}$, permeabilidad $\mu_{s}$ y permisividad relativa $\varepsilon_{r}$.

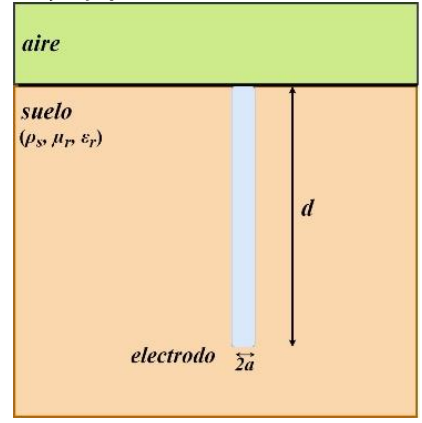

Fig. 1. Electrodo vertical enterrado en un suelo homogéneo.

En la Fig. 2, se muestra una representación genérica del conductor y sus parámetros eléctricos para un segmento $\Delta \mathrm{x}[9]$.
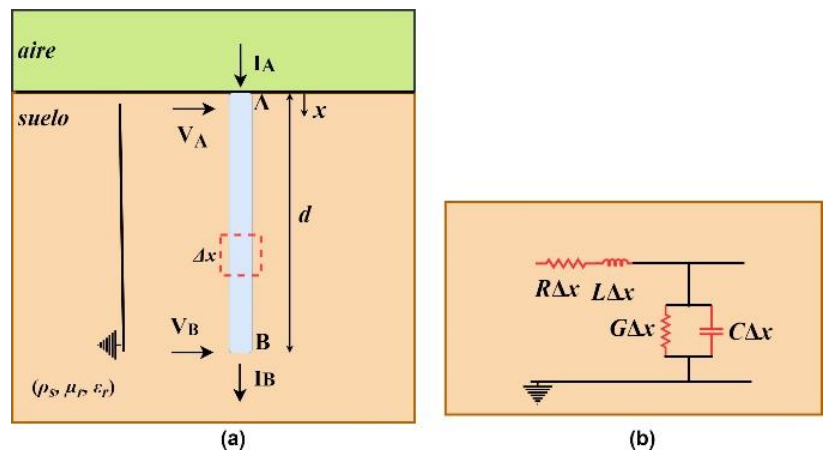

(b)

Fig. 2. Electrodo vertical enterrado en un suelo homogéneo: (a) representación como una línea de transmisión y (b) parámetros eléctricos distribuidos para un segmento $\Delta x$.

Del MLT, el electrodo puede ser visto como una línea de transmisión (LT) corta donde sus parámetros eléctricos son distribuidos por unidad de longitud.

Las variables $V_{A}$ y $I_{A}$ son la tensión y la corriente en el terminal emisor (parte superior del electrodo), mientras que $V_{B}$ y $I_{B}$ son la tensión y corriente en el terminal receptor (parte inferior). En la Fig. 2b, los parámetros en serie $R$ y $L$ son la resistencia e inductancia por unidad de longitud de la línea, respectivamente. Los parámetros transversales $G$ y $C$ son la conductancia y la capacitancia por unidad de longitud de la línea, respectivamente.

Del MLT, para una LT en un suelo conductor posicionado a lo largo del eje $x$, la tensión $V$ y la corriente $I$, en cualquier position $x$, se pueden calcular, en el dominio de frecuencia, como [10]: 
$\frac{\partial^{2} V(x, s)}{\partial x^{2}}=Z Y V(x, s)$

$\frac{\partial^{2} I(x, s)}{\partial x^{2}}=Z Y I(x, s)$

Donde $Z=R+j \omega L$ es la impedancia longitudinal de la línea, $Y=G+j \omega C$ es la admitancia transversal de la línea y $s$ es la frecuencia angular compleja $(s=j \omega)$. Las soluciones de (1) y (2), en el dominio de la frecuencia, para un electrodo de longitud $d$ y considerando que el extremo receptor está en circuito abierto $\left(I_{B}=0\right)$, es dada por las siguientes expresiones hiperbólicas [10]:

$V_{A}(s)=\cosh (\gamma(s) d) V_{B}(s)$

$I_{A}(s)=\frac{1}{Z_{C}} \sinh (\gamma(s) d) V_{B}(s)$

Al dividir (3) y (4), obtenemos la impedancia a tierra, denominada de impedancia harmónica, $Z_{h}(s)$ del electrodo y es dado por [8], [11]:

$Z_{h}(s)=Z_{C} \operatorname{coth}(\gamma(s) d)$

Donde las variables $\gamma(s)$ y $Z_{c}(s)$ son la función de propagación y la impedancia característica de una línea de transmisión dada por [8]:

$$
\begin{aligned}
& \gamma(s)=\sqrt{(R+s L)(G+s C)} \\
& Z_{C}(s)=\sqrt{\frac{(R+s L)}{(G+s C)}}
\end{aligned}
$$

En la literatura [8], [11], [14], muchos autores han propuesto diferentes enfoques de parámetros distribuidos para representar electrodos de conexión a tierra. Basado en la Fig. 2, para el electrodo vertical, los parámetros eléctricos $R, L, G$ y $C$ son dados por [8]:

$$
\begin{aligned}
& R=\frac{\rho_{c}}{\pi a^{2}} \\
& L=\frac{\mu_{c}}{2 \pi}\left[\ln \left(\frac{2 d}{a}\right)-1\right] \\
& C=2 \pi \varepsilon_{r}\left[\ln \left(\frac{4 d}{a}\right)-1\right]^{-1} \\
& G=\frac{1}{\rho_{c} \varepsilon_{r}} C
\end{aligned}
$$

Donde $\rho_{c}$ es la resistividad del conductor.

\section{DEPENDENCIA DE LA FRECUENCIA EN LOS PARAMETROS DEL SUELO}

Según los datos experimentales obtenidos de las mediciones de laboratorio y de campo, la resistividad del suelo $\rho_{s}$ y la permisividad relativa $\varepsilon_{r}$ presentan una fuerte variación a lo largo del rango de DOI: https://doi.org/10.21754/tecnia.v30i2.789 frecuencia típico de las corrientes de rayos (DC a pocos $\mathrm{MHz}$ ) [5]. La permeabilidad del suelo, en general, se puede suponer constante e igual a la permeabilidad al vacío $\mu_{0}$. Para modelar la dependencia de la frecuencia de los parámetros eléctricos del suelo, diversos investigadores han propuesto formulaciones desde la década de 1960 [12]. Con base en los resultados experimentales, desarrollaron expresiones que son capaces de predecir la variación aproximada tanto de la resistividad del suelo $\rho_{s}$ como de la permitividad $\varepsilon_{r}$ en el rango de frecuencia de interés para las corrientes impulsivas (descargas atmosféricas) [5]. En este estudio, se emplean las ecuaciones propuestas por Visacro y Alípio [6], [7].

$$
\begin{aligned}
& \rho=\rho_{0}\left\{1+\left[1.2 \times 10^{-6} \cdot \rho_{0}^{0.73}\right] \cdot\left[(f-100)^{0.65}\right]\right\}^{-1} \\
& \varepsilon_{r}=7.6 \times 10^{3} f^{-0.4}+1.3
\end{aligned}
$$

Donde $\rho_{0}$ es la resistividad del suelo a $100 \mathrm{~Hz}, \rho$ y $\varepsilon_{r}$ son la resistividad del suelo y la permitividad relativa a la frecuencia $f$, respectivamente. La importancia de tener en cuenta la dependencia de la frecuencia en los parámetros del suelo consiste en una disminución significativa de los picos de tensiones producidos por las descargas atmosféricas en comparación con suelos a parámetros constantes [13].

\section{REPRESENTACIÓN POR MEDIO DE CIRCUITOS ELECTRICOS DE LOS SISTEMA DE ATERRAMIENTO}

Los sistemas de aterramiento se pueden representar por medio de circuitos eléctricos concentrados, empleando la técnica del Vector Fitting (VF), que se basa en el método de mínimos cuadrados [14]. Así, el VF ajusta la curva de impedancia del electrodo vertical $Z_{h}(s)$ por una curva aproximada donde los parámetros eléctricos son conocidos. Del método de VF se obtiene una función racional $Z_{h, f i t}(s)$ que representa la curva aproximada. Esta función racional, por su vez, está formada por una suma de fracciones parciales, conforme la ecuación (10).

$Z_{h}(s) \approx Z_{h, f i t}(s)=\sum_{i=1}^{N}\left(\frac{c_{i}}{s-a_{i}}\right)+D+s E$

Donde $\mathrm{N}$ es el número de polos, $c_{i}$ y $a_{i}$ son el residuo y el polo, respectivamente. Los términos $D$ y $E$ son constantes. Las fracciones racionales generadas por el método de VF pueden tener los residuos y polos reales o conjugados complejos que son representados por una combinación de elementos RLC.

Cada fracción racional se puede representar mediante una rama de circuito compuesta por una combinación de elementos RLC. Cuando la fracción racional tiene un residuo real y un polo, el circuito $R L$ 
serie es puede ser empleado. Cuanto los polos y residuos son complexos conjugados, una posible representación es obtenida por el circuito $R L(R / / C)$ [14]. El circuito resultante de la impedancia de aterramiento es formado por $n$ ramas de circuito $R L$ con $m$ ramas de circuito $R L(R / / C)$, donde se verifica que $N=n+m$. El circuito resultante se muestra en la Fig. 3 .

La cantidad de fracciones racionales generadas por el método de VF depende del número de polos $\mathrm{N}$ elegidos para ajustar las curvas. Generalmente, cuantas más oscilaciones presenta la curva de admitancia, mayor es el número de polos necesarios para ajustar la función racional. Sin embargo, aumentar el número de polos no siempre resulta en un mejor ajuste [8].

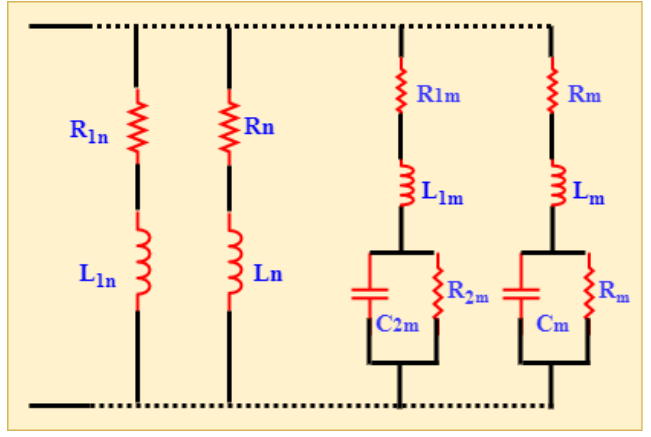

Fig. 3. Circuito eléctrico representativo para sintetizar a impedancia de aterramiento empleando la técnica del Vector Fitting (VF).

\section{RESULTADOS}

Los resultados son divididos en tres secciones. En la sección 5.1, la impedancia del conductor vertical es calculada llevando en consideración el suelo representado por parámetros constantes y dependientes de la frecuencia. En la sección 5.2, es calculado la elevación de potencial en el suelo debido a una descarga atmosférica incidente en el conductor vertical para los dos tipos de suelo. Por fin, en la sección 5.3, las tensiones en los brazos y base de la torre de transmisión son calculadas para una descarga atmosférica incidente en la parte superior de la torre, para los distintos suelos.

\subsection{IMPEDANCIA DEL ELECTRODO VERTICAL CON Y SIN EFECTO DE LA FRECUENCIA}

Las impedancias de aterramiento de un electrodo vertical son calculadas por MLT en función de la frecuencia para diferentes resistividades del suelo. Basado en la Fig. 1, los parámetros considerados son: $d$ $=10 \mathrm{~m}, a=12.5 \mathrm{~mm} \mu_{0}=0.4 \mu \mathrm{H} / \mathrm{m}$. Las resistividades del suelo empleadas son 100, 500 y $1000 \Omega . m$ y el efecto de la frecuencia es computado empleando la ecuación (9). La permisividad de 10 es adoptada para el suelo a parámetros constantes. Las impedancias son DOI: https://doi.org/10.21754/tecnia.v30i2.789 computadas para un rango de frecuencia de $100 \mathrm{~Hz}$ hasta $10 \mathrm{MHz}$, lo que comprende las principales descargas atmosféricas usualmente aplicadas en los estudios de transitorios. La comparación de las impedancias de aterramiento del electrodo vertical con y sin la dependencia de la frecuencia en los parámetros del suelo son ilustradas en la Fig. 4. En las bajas frecuencias, se nota que las impedancias tienen el comportamiento puramente resistivo y la magnitud dependen la resistividad del suelo. A partir de una dada frecuencia, la impedancia puede asumir un comportamiento inductivo o capacitivo predominante en cada rango de frecuencia.

Cuando se considera la dependencia de la frecuencia en los parámetros de suelo, se nota una reducción significativa en la magnitud de la impedancia a partir de una dada frecuencia. Además, esta reducción

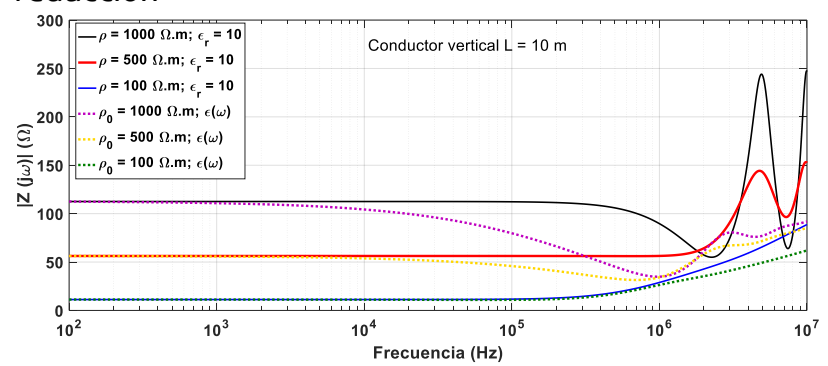

(a)

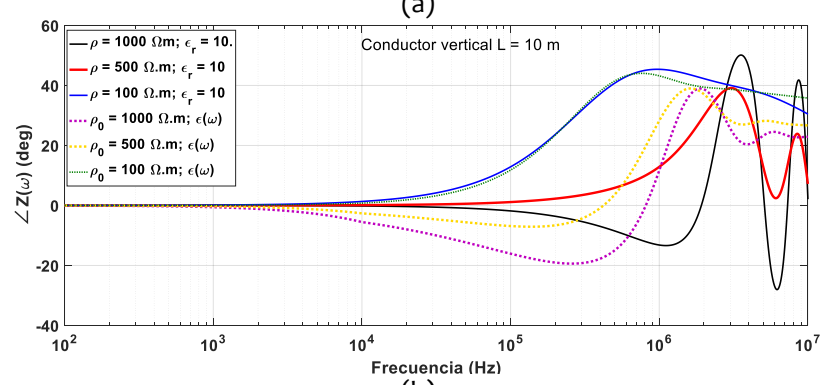

(b)

Fig. 4. Impedancia de aterramiento del conductor vertical para las resistividades de 1.000, 500 y $100 \Omega$.m: (a) Magnitud y (b) fase.

es más pronunciada cuanto mayor es la resistividad de suelo. Esas diferencias en las magnitudes y fases de la impedancia implicaron en modificaciones pronunciadas en las curvas de la elevación del potencial (GPR).

\subsection{CALCULO DEL GPR PARA EL ELETRODO VERTICAL}

El GPR es definido como el producto de la corriente impulsiva y la impedancia del electrodo, siendo el parámetro más importante para seguridad de personas en/o próximas de las subestaciones eléctricas o torres de transmisión. Para el cálculo de GPR, la descarga atmosférica es modelada por una fuente de corriente impulsiva presentada por la función de Heidler, dada en la ecuación (11). 
$i(t)=\frac{I_{0}}{\eta} \frac{\left(t / \tau_{1}\right)^{n}}{1+\left(t / \tau_{1}\right)^{n}} e^{\left(-t / \tau_{2}\right)}$

Dónde lo es la amplitud actual, $\tau_{1}$ es la constante de tiempo frontal, $\tau_{2}$ es la constante de tiempo de cola, $n$ es un exponente con un valor entre 1 y $10, \eta$ es un factor de corrección. Para las simulaciones, los parámetros de la descarga son: $\mathrm{I}_{0}=28 ; \tau_{1}=1.80 \mu \mathrm{s}, \tau_{2}=$ $95 \mu \mathrm{s}, \mathrm{n}=2$ y $\eta=0.823$ y se ilustra la corriente en la Fig. 5.

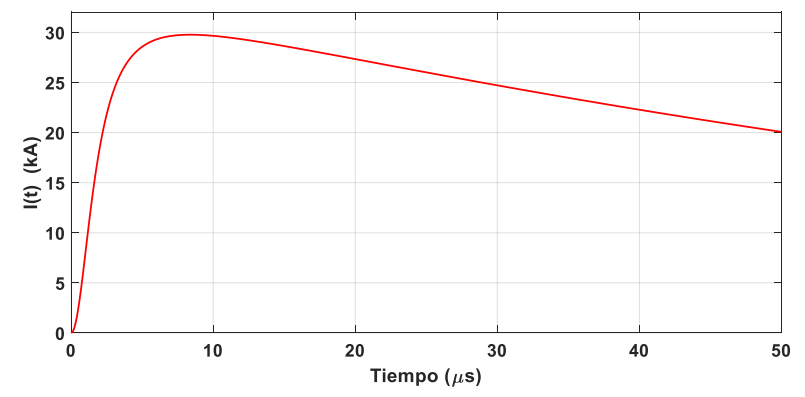

Fig. 5. Corriente impulsiva que representa la descarga atmosférica (First stroke) dada por la función de Heidler.

Los circuitos que sintetizan las impedancias de aterramiento del conductor obtenidas para suelos con parámetros constantes y dependientes de la frecuencia se muestran en la Fig. 6. Se observa que cuando hay la dependencia de la frecuencia en los parámetros del suelo, el circuito eléctrico equivalente tiende a ser más complejo (más ramas para aproximar la impedancia harmónica). Para el cálculo de los GPRs, la impedancia harmónica fue aproximada por $\mathrm{N}=6$ y 8 polos para los ajustes de las impedancias computadas con los parámetros del suelo constantes y dependientes, respectivamente. Estos valores se muestran estables para el VF, donde las curvas de impedancia presentan puntos de inflexión (curvas suaves), lo que garantiza estabilidad de convergencia y los errores entre las curvas original y la aproximada son despreciables. Para el conductor vertical de la Fig. 1 $(\mathrm{d}=10 \mathrm{~m}, \mathrm{a}=12.5 \mathrm{~mm}$ ), los GPRs obtenidos con parámetros constantes y dependientes de frecuencia del suelo son mostrados en la Fig. 7. Se notan que los picos de los GPRs son reducidos para los suelos con efecto de la frecuencia en sus parámetros eléctricos (líneas de trazos). La diferencia es más pronunciada cuando mayor la resistividad del suelo, siendo un importante factor a ser incluido en los análisis de transitorios.

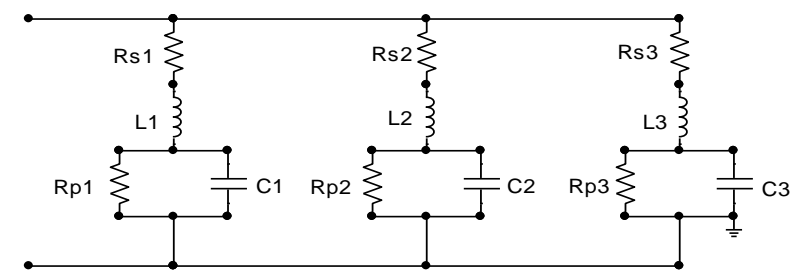

(a)

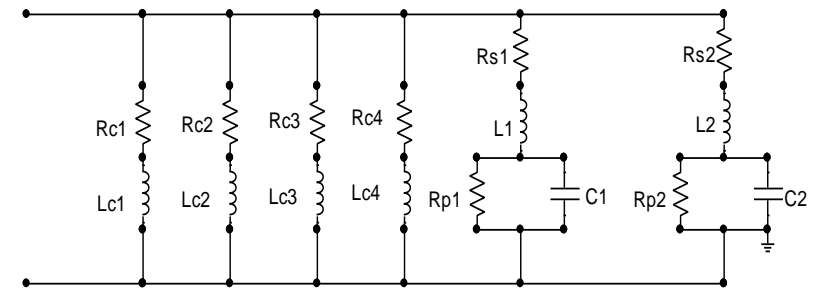

(b)

Fig.6. Circuitos discretos que aproximan la impedancia del electrodo vertical para el suelo: (a) parámetros constantes y (b) parámetros dependientes de la frecuencia.

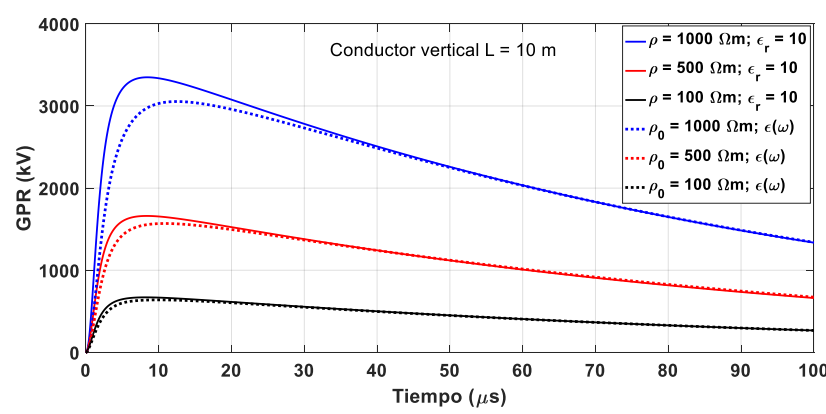

Fig.7. GPRs para el conductor vertical considerando en suelo con parámetros constantes (líneas solidas) y parámetros dependientes de la frecuencia (líneas de trazos).

\subsection{CALCULO DE TENSIONES EN LA TORRE DE TRANSMISIÓN}

Las tensiones en los brazos de una torre de transmisión son calculadas para una descarga atmosférica incidente en la parte superior de la torre. Un conductor vertical de $d=10 \mathrm{~m}$ y $a=12.5 \mathrm{~mm}$ es conectado en su base. El circuito representativo de la torre es mostrado en la Fig. 8. Cada sección está compuesta por una LT corta caracterizada por una impedancia característica $Z_{\mathrm{T}}$, y un circuito $\mathrm{RL}$ paralelo [15]. Las longitudes de cada sección de la torre son: h1 $=8.6 \mathrm{~m}, \mathrm{~h}_{2}=8.50 \mathrm{~m}, \mathrm{~h}_{3}=8.0 \mathrm{~m}$ y $\mathrm{h}_{4}=20 \mathrm{~m}$. Las impedancias características son: $Z_{T_{1}}=\ldots=Z_{T_{4}}=120 \Omega$, velocidad de propagación de $300000 \mathrm{~km} / \mathrm{s}$. Las resistencias $R 1=6.2, R 2=10.6, R 3=10, R 4=26.8 \Omega$ y las inductancias son: $L 1=1.90, L 2=3.20, L 3=3.0$ y $L 4=8.0$ $\mu \mathrm{H}$. La impedancia del canal de la descarga $Z_{g}=400 \Omega$. Las impedancias harmónicas, para una resistividad de suelo de $500 \Omega$.m, fueran aproximadas por $N=6$ y 8 polos para los ajustes de las impedancias del electrodo de $10 \mathrm{~m}$ con y sin efecto de la frecuencia en los parámetros del suelo, conforme mostrado en la Fig. 6. 


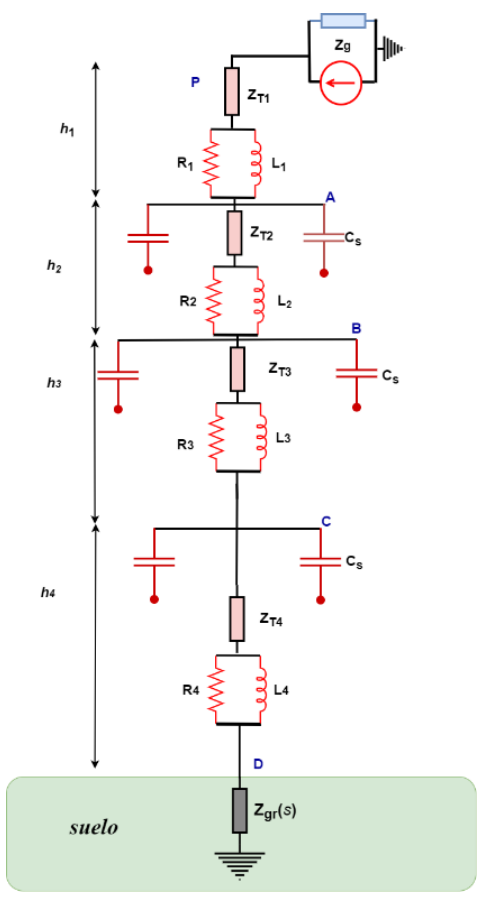

Fig. 8. Torre de transmisión empleada para las simulaciones.

Las tensiones en los puntos $P, A, B, C$ y $D$ son ilustrados en la Fig. 9, para el suelo con parámetros constantes (9a) y con el efecto de la frecuencia (9b). Se observa que las formas de onda, y las magnitudes de los picos, son distintas para los dos casos.

Las tensiones presentan tiempos distintos de propagación, pues son modelados como LT cortas, donde el surto de tensión llega en la base de torre en $0.15 \mu \mathrm{s}$. A partir de ese instante, una parte del surto es absorbido por la impedancia de aterramiento y la otra parte es reflejada para la parte superior, lo que modifica la forma de onda de tensión.

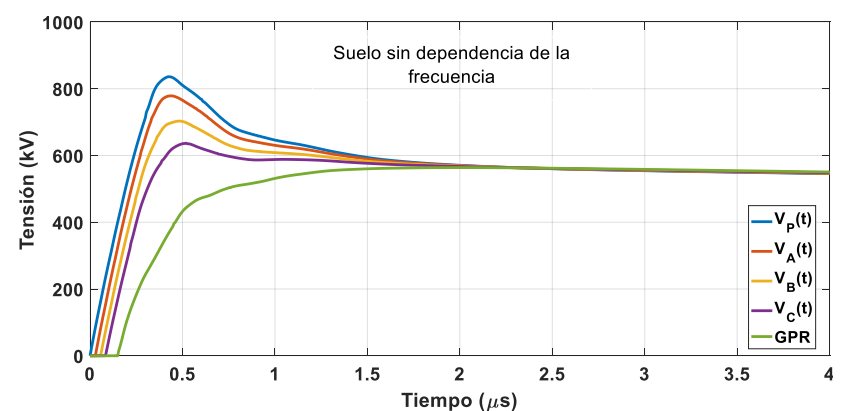

(a)

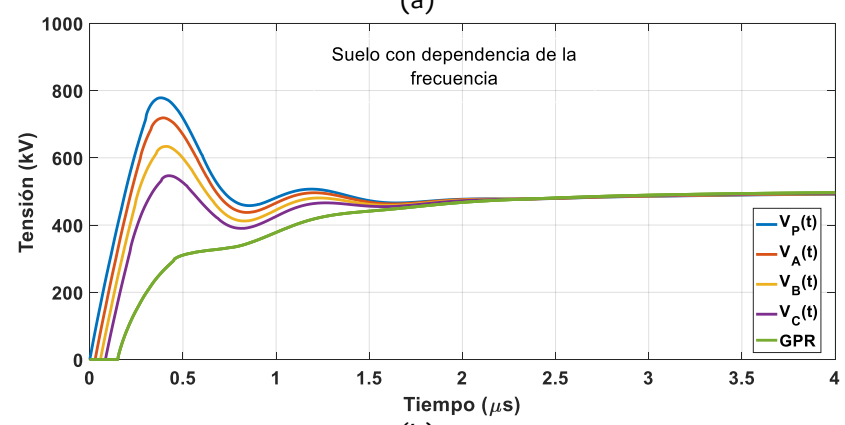

(b)
Fig. 8. Voltajes en los brazos y base de la torre para suelos: (a) con parámetros constantes y (b) dependientes de la frecuencia.

En la tabla I, se observa los picos de las tensiones en los brazos y la base de la torre de transmisión. La diferencia entre los picos cuando se incorpora el efecto de la frecuencia es de $6.92 \%, 7.72 \%, 9.86 \%, 14.15 \%$ y $15,41 \%$, respectivamente para los puntos $P, A, B, C$ y $D$ (GPR), en relación a los picos a parámetros constantes del suelo. Este último, presenta la diferencia más pronunciada, pues parte de la energía inyectada será absorbida por la impedancia de aterramiento, donde el proceso de polarización contribuye para el aumento de la conductividad del suelo cuando el efecto de la frecuencia es considerado.

TABLA I

Picos de las tensiones $(\mathrm{kV})$ en los brazos y base de la torre con y sin efecto frecuencia en los parámetros del suelo.

\begin{tabular}{ccc}
\hline & $\begin{array}{c}\text { Parámetros } \\
\text { constantes }\end{array}$ & $\begin{array}{c}\text { Parámetros dep. } \\
\text { frecuencia }\end{array}$ \\
\hline $\mathbf{V}_{\mathbf{P}}(\mathbf{t})$ & 835.91 & 778.05 \\
$\mathbf{V}_{\mathbf{A}}(\mathbf{t})$ & 778.60 & 718.45 \\
$\mathbf{V}_{\mathbf{B}}(\mathbf{t})$ & 703.14 & 633.8 \\
$\mathbf{V}_{\mathbf{C}}(\mathbf{t})$ & 636.77 & 546.61 \\
$\mathbf{G P R}$ & 559.86 & 473.53 \\
\hline
\end{tabular}

\section{CONCLUSIONES}

Este trabajo ha presentado como la impedancia de aterramiento de un conductor vertical puede presentar un comportamiento variable ente resistivo, inductivo o capacitivo dependiendo del rango de la frecuencia y de los parámetros del suelo. Estos comportamientos deben ser considerados para un análisis preciso. Además, cuando se tiene en cuenta la dependencia de la frecuencia, se observa una reducción significativa en la impedancia de aterramiento a partir de una dada frecuencia, así como, los picos de los GPRs son menores que los obtenidos para suelos a parámetros constantes. Por fin, las tensiones transitorias en diferentes partes de la torre de transmisión están fuertemente modificadas, especialmente en los valores picos, cuando el efecto de la frecuencia en los parámetros del suelo es considerado. Conforme se muestra en la Tabla I, se reducen los picos y su forma de onda de tal modo que esa diferencia pronunciada pueda afectar significativamente los análisis transitorios en líneas de transmisión y este efecto debe ser considerado para proyectos más precisos en los sistemas eléctricos.

\section{AGRADECIMIENTOS}

Los autores agradecen a la Coordenação de Aperfeiçoamento de Pessoal de Nível Superior (CAPES) 
código de financiamento 001, al Conselho Nacional de Desenvolvimento (CNPq) y la Fundação de Amparo a Pesquisa do Estado de São Paulo (FAPESP) proyecto 2019/01396-1 por el soporte económico durante la realización de este trabajo.

\section{REFERENCIAS}

[1] S. A. Halin, A. H. A. Bakar, H. A. Illias, N. H. Hassan, H. Mokhlis, and V. Terzija, "Lightning backflashover tripping patterns on a 275/132 kV quadrupule circuit transmission line in Malaysia", IET Science Measurement \& echnology. , vol. 10, no. 4, pp. 344-354, 2016.

[2] V. Kostic and N. Raicevic, "Grounding system impedance measurement using shifted frequency method", IEEE 5th International Conference on Power Engineering, Energy and Electrical Drives, Riga, 2015, pp. 197-200, 2015.

[3] F. M. Gatta, A. Geri, S. Lauria, and M. Maccioni, "Generalized picircuit tower grounding model for direct lightning response simulation", Electric Power Systems Research, vol. 116, pp. 330-337, 2014.

[4]P. Yadee and S. Premrudeepreechacharn, "Analysis of Tower Footing Resistance Effected Back Flashover Across Insulator in a Transmission System", International Conference Power Sysem. Transients, France, 2007.

[5] H. Karami and K. Sheshyekani, "Harmonic Impedance of Grounding Electrodes Buried in a Horizontally Stratified Multilayer Ground: A Full-Wave Approach", IEEE Transactions on Electromagnetic Compatibility, vol. 60, no. 4, pp. 899-90, 2018.

[6] R. Alipio and S. Visacro, "Frequency dependence of soil parameters: Effect on the lightning response of grounding electrodes", IEEE Transactions on Electromagnetic Compatibility, vol. 55, no. 1, pp. 132-139, 2013.

[7] R. Alipio and S. Visacro, "Modeling the frequency dependence of electrical parameters of soil", IEEE Transactions on Electromagnetic
Compatibility, vol. 56, no. 5, pp. 1163-1171, 2014

[8] L. Grcev and M. Popov, "On high-frequency circuit equivalents of a vertical ground rod", IEEE Transaction Power Delivery, vol. 20, no. 2 II, pp. 1598-1603, 2005.

[9] L. Yang, G. N. Wu, and X. Bin Cao, "An optimized transmission line model of grounding electrodes under lightning currents", Science China Technological Science, no 56. pp. 335-341, 2013.

[10] O. Ramos-Leaos, J. Luis, and J. Alberto, "An Advanced Transmission Line and Cable Model in Matlab for the Simulation of Power-System Transients", MATLAB - A Fundamental Tool for Scientific Computing and Engineering Applications, 1st ed., Rijeka: InTech, Ch. 12, pp. 269-304, 2012.

[11] C. E. F. Caetano, R. Batista, J. O. S. Paulino, W. C. Boaventura, I. J. S. Lopes, and E. N. Cardoso, "A simplified method for calculating the impedance of vertical grounding electrodes buried in a horizontally stratified multilayer ground", in 34th International Conference on Lightning Protection, ICLP 2018, 2018.

[12] D. Cavka, N. Mora, and F. Rachidi, "A Comparison of frequencydependent soil models: Application to the analysis of grounding systems", IEEE Transactions on Electromagnetic Compatibility, vol. 56, no. 1, pp. 177-187, 2014.

[13] S. Piliškić, I. Uglešić, and B. Jurišić, "Evaluating the overvoltage performance of an overhead line taking into account the frequencydependence of its tower's grounding electrodes with high soil resistivity", International Journal of Electrical Power \& Energy Systems, vol. 116, pp. 105547, 2020.

[14] G. Antonini, "SPICE equivalent circuits of frequency-domain responses", IEEE Transactions on Electromagnetic Compatibility, vol. 45, no. 3, pp. 502-512, 2003.

[15] Z. G. Datsios and P. N. Mikropoulos, "Effect of tower modeling on the minimum backflashover current of overhead transmission lines," in the 19th International Symposium on High Voltage Engineering, Czech Republic, 2015. 